CERN-TH.6373/92

TUW-92-1

\title{
ON THE CLASSIFICATION OF QUASIHOMOGENEOUS FUNCTIONS
}

\author{
Maximilian KREUZER冈 \\ Theory Division, CERN \\ CH-1211 Geneva 23, SWITZERLAND \\ and \\ Harald SKARKE曲 \\ Institut für Theoretische Physik, Technische Universität Wien \\ Wiedner Hauptstrasse 8-10, A-1040 Wien, AUSTRIA
}

\begin{abstract}
We give a criterion for the existence of a non-degenerate quasihomogeneous polynomial in a configuration, i.e. in the space of polynomials with a fixed set of weights, and clarify the relation of this criterion to the necessary condition derived from the formula for the Poincaré polynomial. We further prove finiteness of the number of configurations for a given value of the singularity index. For the value 3 of this index, which is of particular interest in string theory, a constructive version of this proof implies an algorithm for the calculation of all non-degenerate configurations.
\end{abstract}

CERN-TH.6373/92

TUW-92-1

January 1992

*e-mail: kreuzer@cernvm.cern.ch

\# e-mail: hskarke@email.tuwien.ac.at 


\section{Introduction}

Recently a particular class of singularities [1, 2], namely singularities of holomorphic quasihomogeneous functions, have been found useful for the classification of superconformal field theories (SCFT) with particular significance for the case of $N=2$ superconformal symmetry [3, 4] due to a non-renormalisation theorem. The requirement of conformal invariance implies quasihomogeneity of degree 1 for the superpotential

$$
W\left(\lambda^{n_{i}} \Phi_{i}\right)=\lambda^{d} W\left(\Phi_{i}\right)
$$

in the effective Lagrangian description, with the scaling dimensions of the chiral superfields $\Phi_{i}$ translating into the weights $q_{i}=n_{i} / d$ of the variables $X_{i}$ of a holomorphic function $W\left(X_{i}\right)$. The local algebra of this function, i.e. the quotient of the polynomial ring by the ideal generated by the gradients $\partial_{j} W\left(X_{i}\right)$, is isomorphic to the operator product algebra of chiral primary states [四]. In order that this algebra be finite we will concentrate on isolated singularities, i.e. require that the origin be the only solution to the equation $d W=0$. We will consider only quasihomogeneous holomorphic functions with positive weights $q_{i}>0$, which are automatically polynomials.

In the mathematical literature isolated singularities have been classified up to 3 variables and for low values of the modality or of the dimension of the local algebra [2]. A general tool for the investigation of degeneracy is the Poincaré polynomial $P(t)=\operatorname{tr} t^{d J}$ [2, 5], where the trace extends over a basis of the local algebra and $J=\sum_{i} q_{i} X_{i} \partial_{i}$ gives the weight of a basis monomial, i.e. the coefficients $\mu_{a}$ of $t^{d q_{a}}$ in this polynomial are the multiplicities of the weights $q_{a}$. For any non-degenerate quasihomogeneous function this polynomial can be calculated from

$$
P(t)=\prod_{i} \frac{1-t^{d-n_{i}}}{1-t^{n_{i}}} .
$$

Thus a necessary condition for non-degeneracy is that this expression is a polynomial with nonnegative coefficients. Note that the Poincaré polynomial only depends on the set of weights and not on the particular form of $W$. We call the set of all polynomials which are quasihomogenous with respect to the weights $q_{i}=n_{i} / d$ a configuration

$$
\mathbb{C}_{\left(n_{1}, \ldots, n_{I}\right)}[d]
$$

A configuration is said to be degenerate if it has only degenerate members. A final guidance of our interest comes from the relation between the singularity index

$$
\hat{c}=\sum_{i}\left(1-2 q_{i}\right)
$$

and the central charge $\mathrm{c}$ in the Virasoro subalgebra of the corresponding $\mathrm{N}=2$ SCFT, which can be shown to be $c=3 \hat{c}$ [4]. 


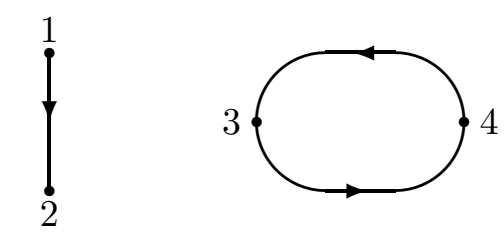

Fig. 1: Graphic representation of $X_{1}^{\alpha_{1}} X_{2}+X_{2}^{\alpha_{2}}+X_{3}^{\alpha_{3}} X_{4}+X_{4}^{\alpha_{4}} X_{3}$.

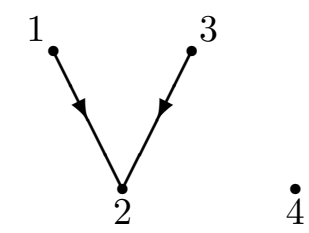

Fig. 2: Graphic representation of the degenerate polynomial $X_{1}^{\alpha_{1}} X_{2}+X_{2}^{\alpha_{2}}+X_{3}^{\alpha_{3}} X_{2}+X_{4}^{\alpha_{4}}$.

In this note we will supply tools for the classification of non-degenerate configurations with a given singularity index. In section 2 we start with some definitions and examples and then give a criterion for non-degeneracy of a configuration. We also show how this criterion is related to the necessary condition that the r.h.s. of (2) is a polynomial (PP-condition). In section 3 we prove that for any given value of the singularity index there is only a finite number of nondegenerate configurations. Then we consider the cases of low integer index $\hat{c} \leq 3$ more explicitly. In the conclusion we summarize our results and briefly comment on their implications for the classification of SCFTs.

\section{Non-degeneracy criterion}

A necessary condition for a quasihomogeneous polynomial to be non-degenerate is that every variable $X$ occurs either in the form $X^{\alpha}$ or $X^{\alpha} Y$. We will use a graphic representation in which every variable is represented by a dot, and a term of the form $X^{\alpha} Y$ is indicated by an arrow from $X$ to $Y$. We will sometimes say ' $X$ points at $Y$ '. If no arrow originates from a variable $X$, then there is a term of the form $X^{\alpha}$ in the polynomial. For example, a polynomial of the form $X_{1}^{\alpha_{1}} X_{2}+X_{2}^{\alpha_{2}}+X_{3}^{\alpha_{3}} X_{4}+X_{4}^{\alpha_{4}} X_{3}$ is shown in Fig. 1.

A possible danger for the non-degeneracy of the polynomial arises when two or more arrows end at the same point, such as in the polynomial $X_{1}^{\alpha_{1}} X_{2}+X_{2}^{\alpha_{2}}+X_{3}^{\alpha_{3}} X_{2}+X_{4}^{\alpha_{4}}$, depicted in Fig. 2. The reason for the problem is that when we calculate $d W / d X_{i}$ and set $X_{2}$ to 0 we are left with 

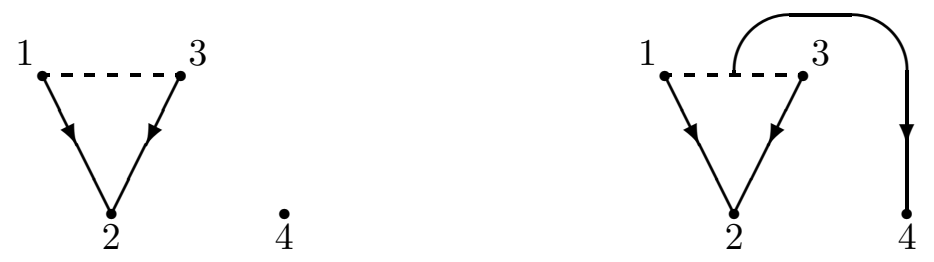

Fig. 3: Dressing of the graph in Fig. 2: (a) by the link $X_{1}^{p} X_{3}^{q}$ and (b) by the pointer $X_{1}^{p} X_{3}^{q} X_{4}$.

only one equation for the two variables $X_{1}$ and $X_{3}$. Therefore we have to introduce extra terms in $W$ that yield terms in $X_{1}$ and $X_{3}$ in $d W$ even when all other variables are set to zero, as in $X_{1}^{\alpha_{1}} X_{2}+X_{2}^{\alpha_{2}}+X_{3}^{\alpha_{3}} X_{2}+X_{4}^{\alpha_{4}}+\varepsilon X_{1}^{p} X_{3}^{q}$ or in $X_{1}^{\alpha_{1}} X_{2}+X_{2}^{\alpha_{2}}+X_{3}^{\alpha_{3}} X_{2}+X_{4}^{\alpha_{4}}+\varepsilon X_{1}^{p} X_{3}^{q} X_{4}$. The graphic representation of these polynomials is shown in Fig. 3. We use dashed lines and pointers originating from dashed lines to indicate such extra terms. We shall call the graph without these additional lines "skeleton graph". It should be noted that all weights can be calculated only with the knowledge of the skeleton. As we have already mentioned, a necessary condition for the existence of a non-degenerate quasihomogeneous function is that the r.h.s of (2) is actually a polynomial. For configurations with up to three variables, but not in general, this condition is also sufficient and identical to the condition for the existence of the terms represented by dashed lines [2].

Definition: We call a variable $X$ a root if the polynomial $W$ contains a term $X^{a}$. A monomial $Y^{a} Z$ is called a pointer at $Z$. $a$ is called the exponent of $X$ or $Y$, respectively. We recursively define a link between two expressions, which may themselves be variables or links, as a monomial depending only on the variables occurring in these expressions. A link may further be linear in an additional variable $Z$, which does not count as a variable of the link. In this case we say that the link points at $Z$, thus extending the previous definition of a pointer. Of course a specific monomial occurring in $W$ can have more than one interpretation as a link or pointer. Given $W$, we call any graph (not necessarily the maximal one) whose lines allow the above interpretation in terms of monomials in $W$ a graphic representation of $W$.

Theorem 1: For a configuration a necessary and sufficient condition for non-degeneracy is that it has a member which can be represented by a graph with:

1. Each variable is either a root or points at another variable.

2. For any pair of variables and/or links pointing at the same variable $\mathrm{Z}$ there is a link joining the two pointers and not pointing at $Z$ or any of the targets of the sublinks which are joined.

Before proving this theorem, we shall illustrate the ideas on which it is based with some examples. 


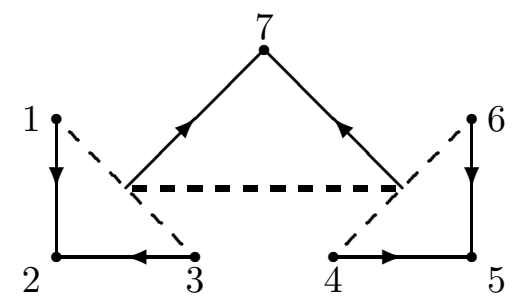

Fig. 4: The pointers $(13) \rightarrow 7$ and $(46) \rightarrow 7$ require the link $((13)(46))$ drawn in boldface.

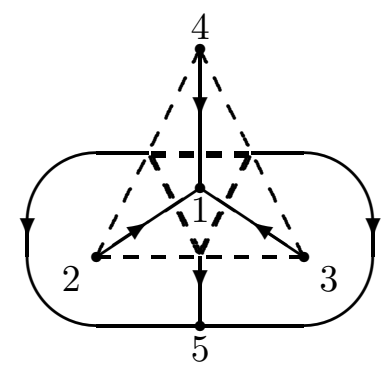

Fig. 5: The monomial $X_{2}^{p} X_{3}^{q} X_{4}^{r}$ provides all three links $((23)(34)),((34)(42))$ and $((42)(23))$ required by the pointers $(23) \rightarrow 5,(34) \rightarrow 5$, and $(42) \rightarrow 5$.

Example 1: A polynomial of the form

$$
X_{1}^{\alpha_{1}} X_{2}+X_{2}^{\alpha_{2}}+X_{2} X_{3}^{\alpha_{3}}+X_{4}^{\alpha_{4}} X_{5}+X_{5}^{\alpha_{5}}+X_{5} X_{6}^{\alpha_{6}}+X_{7}^{\alpha_{7}}+\varepsilon_{1} X_{1}^{p_{1}} X_{3}^{p_{3}} X_{7}+\varepsilon_{2} X_{4}^{p_{4}} X_{6}^{p_{6}} X_{7}
$$

is degenerate, as one can see by calculating $d W$ and setting $X_{2}, X_{5}$ and $X_{7}$ to 0 . By adding the "missing link" $\varepsilon_{3} X_{1}^{q_{1}} X_{3}^{q_{3}} X_{4}^{q_{4}} X_{6}^{q_{6}}$ we obtain non-degeneracy. The translation into our graphic language is given by Fig. 4 .

Example 2: Fig. 5 shows the graphic representation of a polynomial where more than two arrows end at the same point. Distinct "links for links" are realised by the same monomial.

Example 3: $W=X_{1}^{3}+\left(X_{2}^{2}+X_{3}^{2}+X_{4}^{2}\right) X_{1}+\varepsilon X_{2} X_{3} X_{4}$. Note that the last monomial fulfils three tasks at once: pointer from $X_{2}$ and $X_{3}$ to $X_{4}$, pointer from $X_{2}$ and $X_{4}$ to $X_{3}$ and pointer from $X_{3}$ and $X_{4}$ to $X_{2}$.

Proof of Theorem 1: A) 'Necessary': Calculating $d W / d X_{i}$ yields $n$ equations in $n$ variables. Non-degeneracy means that they can be fulfilled only by the trivial solution. Obviously a necessary condition is that by setting $k$ variables to 0 no more than $k$ equations can be fulfilled identically. Starting with $k=n-1$, we find that for each $X_{i}$ monomials in this variable must occur in $d W / d X_{j}$, which means that each variable has to be a root or has to point at another variable. (This was the first condition of the theorem).

Let us now consider $k=n-2$, i.e. we set to 0 all variables except two (which we shall call $X_{1}$ 
and $X_{2}$ ). According to above, monomials $X_{1}^{\alpha_{1}}$ and $X_{2}^{\alpha_{2}}$ must occur. There are two possibilities: either these monomials occur in different equations, meaning that there are at least two equations that are not automatically fulfilled by setting all variables except $X_{1}$ and $X_{2}$ to zero, or they both occur in the same equation. In the latter case a necessary condition for non-degeneracy is the occurrence of a monomial in both variables in one of the other equations, i.e. either one of them points at the other or there must be a link between them.

We proceed inductively in $l=n-k$ : We construct the graph by adding just the lines that we need in each step. Assume we have all the lines up to level $l-1$. Suppose there are two links pointing at the same variable $Z$. Let them have $l_{1}<l$ and $l_{2}<l$ variables, of which they have $q$ variables in common, and $l=l_{1}+l_{2}-q$ variables together. Setting all other variables to zero, the links and pointers we have drawn until now correspond to $l_{1}+l_{2}-q-1=l-1$ equations, as $\partial W / \partial Z$ and $q$ additional equations are double counted due to the overlap in the variables. So we need one more equation in the $l$ variables, i.e. we need the link implied by the second condition of the criterion.

B) 'Sufficient': We show that no degenerate configuration can fulfil our conditions. Let $W=$ $\sum M_{\mu} \varepsilon^{\mu}$ represent a degenerate configuration, where the $M_{\mu}$ are monomials in $X_{i}$, and let $\partial_{i}=$ $\partial / \partial X_{i}$ and $\partial_{\mu}=\partial / \partial \varepsilon^{\mu}$. Further, for any choice of $\varepsilon^{\mu}$ there are non-vanishing solutions $X_{i}(\varepsilon)$ to the equations $\partial_{i} W=0$. As these equations are polynomial, we can choose an open set of $\left(\varepsilon^{1}, \ldots, \varepsilon^{k}\right)$ and a solution $\left(X_{1}(\varepsilon), \ldots, X_{n}(\varepsilon)\right)$ depending smoothly on the $\varepsilon^{\mu}$ in this set. Quasihomogeneity implies that $W\left(X_{i}(\varepsilon)\right) \equiv 0$. Therefore

$$
\frac{d}{d \varepsilon^{\mu}} W=\sum_{i} \partial_{i} W \partial_{\mu} X_{i}+\partial_{\mu} W=M_{\mu}=0,
$$

i.e. every monomial in $W$ has to vanish. We now choose a point $\varepsilon$ in this set for which $X_{1}(\varepsilon) \neq$ $0, \ldots, X_{l}(\varepsilon) \neq 0$, but $X_{l+1} \equiv \ldots \equiv X_{n} \equiv 0$ in some neighbourhood of $\varepsilon$ (we are of course free to choose the labels for the $\left.X_{i}\right)$. Differentiating $\partial_{i} W$ with respect to $\varepsilon^{\mu}$ we find

$$
\frac{d}{d \varepsilon^{\mu}} \partial_{i} W=\sum_{j \leq l} \partial_{i} \partial_{j} W \partial_{\mu} X_{j}+\partial_{i} M_{\mu}=0 .
$$

Due to quasihomogeneity $\sum_{j \leq l} q_{j} X_{j} \partial_{i} \partial_{j} W=\left(1-q_{i}\right) \partial_{i} W=0$, i.e. the rank of the rectangular matrix $\partial_{i} \partial_{j} W$ with $j \leq l$ is less than $l$. Thus there are at least $n-l+1$ independent vectors $c_{i}^{(m)}$ with $\sum_{i \leq n} c_{i}^{(m)} \partial_{i} \partial_{j} W=0$ and hence $\sum_{i \leq n} c_{i}^{(m)} \partial_{i} M_{\mu}=0$. As all monomials $M_{\mu}$ have to vanish, only one of the derivatives $\partial_{i} M_{\mu}$ can be non-vanishing for a given $\mu$. Thus the sum $\sum_{i} c_{i}^{(m)} \partial_{i} M_{\mu}$ has at most one non-vanishing contribution, and, as $c_{i}^{(m)}$ can be 0 for all $m$ for at most $l-1$ values of $i$, all variables $X_{1}, \ldots, X_{l}$ and all links among these variables have to point at a subset of at most $l-1$ variables $X_{j}$ with $j>l$. The resulting double pointer cannot be completely resolved, as the required link would again have to point at the same set of $l-1$ variables and thereby generate a new double pointer. //. 
Lemma 1: The necessary condition for non-degeneracy that the expression (2) for the Poincaré polynomial is a polynomial (we refer to this as the PP-condition) is equivalent to the criterion of theorem 1 if one omits the requirement that all exponents in the link monomials have to be non-negative.

Proof: $\Pi\left(1-t^{d-n_{i}}\right) /\left(1-t^{n_{i}}\right)$ is a polynomial if all zeros in the denominator, counted according to their multiplicities, are matched by zeros in the numerator, i.e. if the set of all multiples of $1 / n_{i}$ between 0 and 1 is a subset of the multiples of $1 /\left(d-n_{i}\right)$, even when multiplicities are taken into account. The relaxed condition on the links, which we are referring to, is that the number theoretic condition $\sum p_{i} n_{i}=d$ (for non-pointing links) or $\sum p_{i} n_{i}=d-n_{k}$ (for a link pointing at $X_{k}$ ), where the sum runs over all $X_{i}$ joined by the link, is fulfilled without requiring that the $p_{i}$ all be non-negative. This is equivalent to the condition that the greatest common divisor of these $n_{i}$ divides $d$ or some $d-n_{k}$. To show the equivalence of these two conditions we first note that the PP-condition implies that each $X_{i}$ has to be a pointer or a root, as $1 / n_{i}$ must be a multiple of some $1 /\left(d-n_{k}\right)$. In this context a root is to be considered as a pointer at itself.

There is a problem, however, if two variables $X_{i}$ and $X_{j}$ point at the same variable $X_{k}$. Then the multiples of $1 /\left(n_{i} \cap n_{j}\right)$ are contained only once and have to be taken care of by some further variable $X_{l}$ with $n_{i} \cap n_{j}$ dividing $d-n_{l}$. We thus recover the requirement of the links implied by the non-degeneracy criterion except for the positivity of the exponents in the monomial. If $l$ cannot be chosen as $i$ or $j$, i.e. if $n_{i} \cap n_{j}$ does not divide $d$, this link is a pointer at $X_{l}$. The roots, considered as pointers at themselves, and the links which are no pointers, i.e. can be considered as pointing at one of their variables, do not imply additional links. This is because the corresponding $n_{i}$ (or $n_{i} \cap n_{j}$ for pointers) are divisors of $d$. In the simplest case, for example, where $n_{i}$ and $n_{j}$ divide $d-n_{i}$, the missing ratio $1 /\left(n_{i} \cap n_{j}\right)$ is a multiple of $1 /\left(d-n_{j}\right)$ and thus acts like a pointer at $X_{j}$ with the additional feature that the denominator divides $d$. In this way the missing numbers can be passed on backwards along any pointers with ever smaller denominators until they find their match in a free multiple of some $1 /\left(d-n_{k}\right)$ with no other pointer at $X_{k}$ or until $n_{i} \cap \ldots \cap n_{k}=1$. Proceeding in the same way with the additional overlaps which may arise due to the additional pointers in each step, we indeed find equivalence of the PP-condition and the relaxed link criterion. //.

To illustrate this equivalence we use the following example by B. M. Ivlev of a degenerate configuration fulfilling the PP-condition [2],

$$
\mathbb{C}_{(1,24,33,58)}[265]
$$

with the corresponding skeleton polynomial

$$
X^{265}+X Y^{11}+X Z^{8}+Z U^{4}
$$


To fulfil the criterion of theorem 1 we would need a link between $Y$ and $Z$, which has to point at $U$ as neither $d$ nor any $d-n_{i}$ is a multiple of $3=24 \cap 33$ except for $d-n_{U}=207=24 p+33 q$. This equation, however, does not have a solution with both $p$ and $q$ positive, which explains why (22) is a polynomial although the configuration is degenerate. It would be interesting to find out if it is possible to construct an $\mathrm{N}=2 \mathrm{SCFT}$ with these weights.

\section{On configurations with a given index}

Lemma 2: If a non-degenerate configuration contains $n$ variables $X_{i}$ with a given weight $q \in$ $(1 / 3,1 / 2)$, then it also contains at least $n$ variables $Y_{j}$ of weight $\bar{q}=1-2 q$.

Proof: Let $W=W\left(X_{i}, Y_{j}, Z_{k}\right)$ with weight $\left(X_{i}\right)=q$, weight $\left(Y_{j}\right)=\bar{q}$ and weight $\left(Z_{k}\right) \neq q, \bar{q}$. We calculate $d W$ and set $Y_{j}$ and $Z_{k}$ to 0 . Non-degeneracy implies that at least $n$ equations for the $X_{i}$ must remain. Because $q \in(1 / 3,1 / 2)$, these equations must be quadratic in the $X_{i}$, i.e. of weight $2 q=1-\bar{q}$. Therefore they must come from $d W / d Y_{i}$, implying that the number of $Y_{i}$ 's is at least n. $/ /$.

We call variables trivial if they correspond to terms $X^{2}$. Trivial variables have weights $q=1 / 2$ and therefore do not contribute to $\hat{c}$, nor to the local algebra, as they can be eliminated by $\partial W / \partial X=0$.

Corollary 1: For every non-degenerate configuration $\hat{c}$ is greater than or equal to $1 / 3$ times the number of non-trivial variables.

Proof: Grouping variables with $q \in(1 / 3,1 / 2)$ together with $\bar{q}$, we have $1-2 q+1-2 \bar{q}=2 q>2 / 3$; the contributions of all other non-trivial variables (with weights $\leq 1 / 3$ ) are at least $1-2 / 3=1 / 3$. $/ /$.

Theorem 2: Given a positive rational number $\hat{c}$, there is only a finite number of non-degenerate configurations whose index is $\hat{c}$.

Proof (indirect): Suppose there is an infinite sequence of configurations with index $\hat{c}$. Due to the above corollary there is only a finite number of skeleton graphs that can realize $\hat{c}$, so there must be an infinite subsequence corresponding to just one graph. Considering a specific exponent, we can find either a subsequence for which this exponent is constant or one for which this exponent goes monotonically to infinity. Doing this for every exponent, we end up with a sequence of polynomials corresponding to the same skeleton graph, for which $n-m$ exponents are constant while $m$ exponents tend monotonically to infinity. Of course the "limit configuration", which contains $m$ variables of weight 0 , also has index $\hat{c}$. We denote the weights in the $l$ 'th member of 
the sequence by $q_{i}^{(l)}, i \in\{1, \cdots, n\}$ with $\lim _{l \rightarrow \infty} q_{i}^{(l)}=0$ for $i \in\{1, \cdots, m\}$. We will now show that the index of a member of such a sequence is in fact smaller than the index of the limit sequence, in contradiction with the assumed constance of $\hat{c}$. Consider a specific $l$ and let $\varepsilon=\max q_{i}^{(s)}$ with $i \leq m$ and $s \geq l$. We define the intervals $I_{k}=\left(2^{-k} \varepsilon, 2^{-k+1} \varepsilon\right]$. By $A_{k}$ we denote the number of points with $q^{(l)}-q^{(\infty)} \in I_{k}$, by $B_{k}$ we denote the number of points with $q^{(\infty)}-q^{(l)} \in I_{k}$. Note that $B_{1}=0$, as for any variable $q^{(\infty)}=0$ or the possible target variable has $q<\frac{1}{2}$. Now consider all $\sum_{i=1}^{k} B_{i}$ points for which $q^{(\infty)}-q^{(l)}>2^{-k} \varepsilon$. Setting all other variables to zero in $d W / d X_{i}$, we see that we need at least as many equations, coming from points with $q^{(l)}-q^{(\infty)}>2^{-k+1} \varepsilon$, as these equations are at least quadratic in the non-zero variables. We thus have $\sum_{i=1}^{k} B_{i} \leq \sum_{i=1}^{k-1} A_{i}$, i.e. $\sum_{i=1}^{k}\left(A_{i}-B_{i+1}\right) \geq 0$ and

$$
\hat{c}^{(\infty)}-\hat{c}^{(l)}=2 \sum\left(q^{(l)}-q^{(\infty)}\right)>2 \varepsilon \sum_{i}\left(2^{-i} A_{i}-2^{-i+1} B_{i}\right)=2 \varepsilon \sum_{i} 2^{-i}\left(A_{i}-B_{i+1}\right) \geq 0 .
$$

This is the contradiction we were looking for. //.

Theorem 3: Given a rational number $r$, one can find a positive number $\varepsilon$ such that no number in the interval $(r, r+\varepsilon)$ is the index of a non-degenerate quasihomogeneous polynomial; i.e. the accumulation points in this set of indices are all approached from below.

Proof: Like the previous theorem with an infinite sequence of configurations with decreasing indices $\hat{c}^{(l)} \rightarrow r$ instead of constant index. //.

Theorem 2 has given us valuable theoretical information, but does not help us in explicitly finding all configurations of a given index. Especially for the case $\hat{c}=3$, which is most important for string theory, one would like to have a way of constructing normal forms for all possible configurations. We will now formulate and prove a lemma which makes it possible to write a computer program which calculates explicitly all configurations with $\hat{c}=3$.

Lemma 3: For a non-degenerate quasihomogeneous polynomial with $\hat{c}=3$ the number of exponents $\alpha_{i}>18$ or $\alpha_{i}>84$ is smaller than 3 or 2 , respectively. These limits cannot be improved, as is seen from the polynomials $X_{1}^{3}+X_{2}^{18}+X_{3}^{18}+X_{4}^{18}$ and $X_{1}^{3}+X_{2}^{7}+X_{3}^{84}+X_{4}^{84}$.

Proof: Let us assume that there are three exponents $\alpha_{i}>18$, corresponding to variables with weights $q_{1}, q_{2}, q_{3}<1 / 18$, contributing $1-2 q_{i}$ to $\hat{c}$. If there are also variables with exponents $\alpha=2$, pointing at variables with $q_{i}<1 / 18$, they add $1-2\left(1 / 2\left(1-q_{i}\right)\right)=q_{i}$ to $\hat{c}$. The total contribution of the variables considered so far is therefore between $3(1-2 / 18)=3-1 / 3$ and 3 . It is impossible to complete this to a polynomial with $\hat{c}=3$. This proves the first assertion.

Assume now that there are terms $X_{i}^{a_{i}}$ or $X_{i}^{a_{i}} Y^{i}$ with $a_{i}>84, i=1,2$ in the potential, thus $q_{i}<1 / 84$. The contribution $\hat{c}_{12}$ of these variables to $\hat{c}$ fulfils $2-\frac{1}{21}<\hat{c}_{12}=2-2 q_{1}-2 q_{2}<2$. A "partner variable" $Z_{i}$ pointing at $X_{i}$ with exponent 2 , i.e. a term $X_{i} Z_{i}^{2}$, can be disregarded as it effectively just doubles $a_{i}$ (if $a_{1}=a_{2}$ both possible partner variables might point to the same 
$\left.X_{i}\right)$. According to lemma 2 there have to be 2 or 3 more variables in order to make up for the difference to a total $\hat{c}$ of 3 .

Case 1: 2 additional variables $U_{i}$ with weights $r_{i}$. If one of their exponents is 2 , it has to point at a $Z_{i}$, since $U_{1}$ and $U_{2}$ have to contribute more than 1 . Then one can explicitly calculate the contribution of $X_{1}, X_{2}, Z_{1},\left(Z_{2},\right) U_{1}$ to be $\frac{5}{2}-\frac{3}{2} q_{1}-(2) q_{2}$. $\hat{c}=3$ would then require $r_{2}=$ $\frac{1}{4}-\frac{3}{4} q_{1}-\left(\frac{1}{2}\right) q_{2}$, which cannot be satisfied. The exponents of $U_{1}, U_{2}$ also have to be less than 7 , since $\frac{1}{3}+1-\frac{2}{7}=\frac{22}{21}$. Enumeration of all relevant singularities in 2 variables shows that the smallest possible contribution to $\hat{c}$ larger than 1 is $22 / 21$, thus the total $\hat{c}$ cannot be 3 . If one or both $U_{i}$ point at some $X_{i}$ or $Z_{i}, \hat{c}$ can only be enlarged. So we finally have to consider the contributions with $\hat{c}=2-2\left(r_{1}+r_{2}\right) \leq 1$. Pointers at $X_{j}$ would not make any difference, as the decrease in $r_{i}$ would be $r_{i} q_{j}$, which is not sufficient to reach $\hat{c}=3$. So let $U_{1}$ point at $Z_{j}$. This makes $1-2 r_{1}$ larger than $\frac{2}{3}-\frac{1}{252}$. Thus $U_{2}$ cannot point at a $Z_{k}$ and its exponent has to be 3 , so $U_{2}$ cannot make up for the difference to $\hat{c}=3$.

Case 2: 3 additional variables $U_{i}$ with weights $r_{i}$. We split this case according to the number of exponents equal to 2. If all exponents are larger than 2 this implies $r_{i} \leq \frac{1}{3}$, and hence $r_{i} \geq \frac{13}{42}=\frac{1}{3}\left(1-\frac{1}{14}\right)$, i.e. all exponents have to be 3 . These variables thus may only point at a variable with weight less than $1 / 14$, i.e. at $X_{i}$. Examining all cases there are 10 infinite series of polynomials with $\hat{c}=3$ :

$$
\begin{array}{lll}
X^{a}+Y^{6 a}+X Z^{2}+U^{3} X+V^{3} X+W^{3} & \text { and } & Y^{6 a} \rightarrow Y^{3 a}+Y T^{2} \\
X^{a}+Y^{4 a}+X Z^{2}+U^{3} X+V^{3} X+W^{3} Y & \text { and } & Y^{4 a} \rightarrow Y^{a}+Y T^{2} \\
X^{a}+Y^{2 a}+X Z^{2}+U^{3} X+V^{3} X+W^{3} X & \text { and } & Y^{2 a} \rightarrow Y^{a}+Y T^{2} \\
X^{a}+Y^{a}+X\left(U^{3}+V^{3}+W^{3}+Z^{2}+T^{2}\right) & \text { and } & X W^{3} \rightarrow Y W^{3} \\
X^{a}+Y^{a}+X\left(U^{3}+V^{3}+W^{3}\right)+Y\left(Z^{2}+T^{2}\right) & \text { and } & X W^{3} \rightarrow Y W^{3}
\end{array}
$$

For none of these polynomials, however, do the links which would be necessary to make them non-degenerate exist for $a>84$. In the first case, for example, it is impossible to have a link between $Z$ and $U$, since the weights of these two variables add up to $\frac{5}{6}\left(1-\frac{1}{a}\right)$.

Now let the first exponent be equal to 2. $U_{1}$ must not point at $Z_{i}$ (otherwise $\hat{c}>3$ ), so it points at $U_{2}$ and the exponents of $U_{2}$ and $U_{3}$ have to be 3 as above. If the latter variables do not point further the total $\hat{c}$ stays below 3 . They might only point at $X_{1}$, because otherwise we would violate $\hat{c} \leq 3$. This, however, does not help either, because the decrease of $r_{2}$ and/or $r_{3}$ is sufficiently compensated by the increase of $r_{1}$ to keep $\hat{c}$ below 3 . The proof is completed by the observation that there cannot be 2 exponents of the $U$ 's equal to 2 , because one of these variables would then have to point at a $Z_{i}$, which would increase $\hat{c}$ beyond 3 for an isolated configuration. $/ /$.

Lemma 3 , together with corollary 1 , implies an algorithm for the calculation of all configurations with $\hat{c}=3$, as the only unconstrained exponent in each skeleton graph can be calculated 
(and of course has to turn out integer to yield a solution). It is straightforward to further reduce the number of possibilities using lemma 2. Consider, for example, a skeleton graph with $n$ points of which $i$ have an exponent larger than or equal to $a$. If $p$ of these $i$ variables are pointed at by a variable with exponent 2 , this implies

$$
\hat{c} \geq(n-i-p) / 3+i(1-2 / a)+p / a
$$

If $n+2 i>3 \hat{c}+p$ this is equivalent to

$$
a \leq 3(2 i-p) /(n+2 i-3 \hat{c}-p)
$$

As $\partial a / \partial p$ is always positive, and obviously $p \leq i$ and $p \leq n-i$, we obtain the bounds $a \leq$ $3 i /(n+i-3 \hat{c})$ or $a \leq(3 i-n) /(i-\hat{c})$ for $i \leq n / 2$ or $i \geq n / 2$, respectively. It is also straightforward to check that not more than $i$ variables can have an exponent larger than $a=2 i /(i-\hat{c})$.

For completeness we state the following results referring to $\hat{c}=1$ or 2 . They can be proved with the same methods as above.

Lemma 4: Any configuration with $\hat{c}=1$ corresponds to weights $(1 / 3,1 / 3,1 / 3),(1 / 3,1 / 6)$ or $(1 / 4,1 / 4)$. For a polynomial with $\hat{c}=2$ there is at most one exponent greater than 12 and no exponent can be greater than 42 .

\section{Conclusion}

We have given a criterion for the non-degeneracy of a configuration which requires the check of a recursive condition concerning the existence of certain monomials consistent with quasihomogeneity, and have introduced a convenient graphic representation for these monomials. The $\mathrm{PP}$-condition is equivalent to this condition except for positivity of the exponents. We have also shown that for a given singularity index the number of non-degenerate configurations is finite and that such a value cannot be approximated from above by non-degenerate configurations. Finally, applications of these results provide the necessary ingredients for explicit calculations of all configurations at least for low values of $\hat{c}$.

Our results in particular imply an algorithm for the calculation of all configurations with $\hat{c}=3$, which is straightforward to implement due to the recursive structure of the condition of theorem 1. According to lemma 2 only polynomials in up to $3 \hat{c}$ non-trivial variables have to be considered. As the weights of all variables are already determined by the skeleton graph, one only has to investigate a reasonable number of such graphs, which can be constructed recursively. The crucial point is that the combinations of exponents which have to be checked can be restricted 
to a finite number even for these skeletons, as has been done in lemma 3 for the particularly interesting case $\hat{c}=3$. Non-degeneracy can then be checked in a second step.

Such a construction would be an extension of the work by Candelas et al. [6] on CalabiYau manifolds in weighted projective spaces [7]. The connection between these manifolds and the construction of $N=2$ SCFT from non-degenerate quasihomogeneous functions has been discussed by Greene et al. in ref. [8]. The formulae for the calculation of the Hodge numbers from the scaling dimensions of the superfields have later been supplied by Vafa [9] and rederived by methods of algebraic geometry in ref. [10]. The authors of ref. [6] have implemented 30 polynomials fulfilling the criterion of theorem 1 and have constructed some 6000 Calabi-Yau manifolds in weighted $\mathbb{P}_{4}$. As the number of skeleton graphs already grows faster than $2^{n}$ for polynomials in $n$ variables, a complete construction along these lines, however, appears to be difficult for larger numbers of variables.

\section{References}

[1] V.I.Arnold, Singularity Theory, London Mathematical Society Lecture Note Series, Vol. 53, Cambridge Univ. Press 1981

[2] V.I.Arnold, S.M.Gusein-Zade and A.N.Varchenko, Singularities of Differentiable Maps, Vol. I, Birkhäuser 1985

[3] E.Martinec, Phys. Lett. B217 (1989) 431;

C.Vafa and N.Warner, Phys. Lett. B218 (1989) 51

[4] W.Lerche, C.Vafa and N.P.Warner, Nucl. Phys. B324 (1989) 427

[5] N.Bourbaki, Lie Groups and Lie Algebras, Hermann, Paris 1971, chapter V, section 5.1

[6] P.Candelas, M.Lynker and R.Schimmrigk, Nucl. Phys. B341 (1990) 383

[7] I.Dolgachev, in: Group Actions and Vector Fields, ed. J.Carrell, Springer Lecture Notes in Mathemathics, Vol. 956 (Springer, Berlin);

A.Dimca, J. Reine ang. Math. 366 (1986) 458

[8] B.R.Greene, C.Vafa and N.P.Warner, Nucl. Phys. B324 (1989) 371

[9] C.Vafa, Mod. Phys. Lett. A4 (1989) 1169; Superstring Vacua, HUTP-89/A057 preprint

[10] S.S.Roan, Int. J. Math. 1 (1990) 211 